\title{
Impact of the number of examined lymph nodes on stage migration in node-negative gastric cancer patients: a Chinese multi-institutional analysis with propensity score matching
}

\begin{abstract}
Pengfei Gu ${ }^{1 \#}$, Jingyu Deng ${ }^{1 \#}$, Wei Wang ${ }^{2 \#}$, Zhenning Wang ${ }^{3 \#}$, Zhiwei Zhou ${ }^{2}$, Huimian Xu ${ }^{3}$, Han Liang ${ }^{1}$
${ }^{1}$ Department of Gastric Cancer, Tianjin Medical University Cancer Institute \& Hospital, National Clinical Research Center of Cancer, Key Laboratory of Cancer Prevention and Therapy, Tianjin's Clinical Research Cancer for Cancer, Tianjin, China; ${ }^{2}$ Department of Gastric and Pancreatic Surgery, Sun Yat-sen University Cancer Center, State Key Laboratory of Oncology in South China, Collaborative Innovation Center for Cancer Medicine, Guangzhou, China; ${ }^{3}$ Department of Surgical Oncology, The First Affiliated Hospital of China Medical University, Shenyang, China

Contributions: (I) Conception and design: H Liang, J Deng, H Xu, Z Zhou; (II) Administrative support: H Liang, J Deng, H Xu, Z Zhou; (III) Provision of study materials or patients: H Liang, J Deng, H Xu, Z Zhou; (IV) Collection and assembly of data: P Gu, J Deng, W Wang, Z Wang; (V) Data analysis and interpretation: P Gu, J Deng, W Wang, Z Wang; (VI) Manuscript writing: All authors; (VII) Final approval of manuscript: All authors.

"These authors contributed equally to this work.

Correspondence to: Han Liang, MD, PhD. Department of Gastric Cancer, Tianjin Medical University Cancer Institute \& Hospital, National Clinical Research Center of Cancer, Key Laboratory of Cancer Prevention and Therapy, Tianjin's Clinical Research Cancer for Cancer, Tianjin 300060, China. Email: tjlianghan@126.com; Zhiwei Zhou, MD, PhD. Department of Gastric and Pancreatic Surgery, Sun Yat-sen University Cancer Center, State Key Laboratory of Oncology in South China, Collaborative Innovation Center for Cancer Medicine, Guangzhou 510060, China. Email: zhouzhw@sysucc.org.cn; Huimian Xu, MD, PhD. Department of Surgical Oncology, The First Affiliated Hospital of China Medical University, Shenyang 110001, China. Email: xuhuimian@126.com; Jingyu Deng, MD, PhD. Department of Gastric Cancer, Tianjin Medical University Cancer Institute \& Hospital, National Clinical Research Center of Cancer, Key Laboratory of Cancer Prevention and Therapy, Tianjin's Clinical Research Cancer for Cancer, Tianjin 300060, China. Email: dengery@126.com.
\end{abstract}

Background: This propensity score matching (PSM) analysis assessed the influence of examined lymph nodes (ELNs) count on stage migration and survival in node-negative (pN0) gastric cancer (GC) patients.

Methods: We performed a retrospective analysis of 7,620 GC patients who underwent curative gastric resection in three Chinese medical centers. PSM was used to reduce the confounding effects between the pN0 GC patients with ELNs $<16$ or $\geq 16$. Survival differences among various subgroups of GC patients were analyzed to assess the impact of the ELNs count on the stage migration in accordance with the overall survival (OS) of pN0 GC patients.

Results: After matching, the backgrounds of pN0 GC patients in the ELNs $<16$ ( $\mathrm{n}=825)$ and ELNs $\geq 16$ $(n=826)$ groups were well-balanced. Survival analyses revealed that the ELNs count was positively correlated with the OS $(\mathrm{P}=0.001)$. Multiple Cox analysis indicated that the ELNs count was an independent predictor of the OS in pN0 GC patients. Stage migrations were mainly detected in subgroups of pN0 GC patients with specific pTNM stages, as follows: (I) pT2N0M0 with ELNs $<16$ vs. pT3N0M0 with ELNs $\geq 16$; (II) pT3N0M0 with ELNs <16 vs. pT3N1M0 with ELNs $\geq 16$; and (III) pT4aN0M0 with ELNs $<16$ vs. pT4aN1M0 with ELNs $\geq 16$.

Conclusions: We show that stage migration can be detected in pN0 GC patients, and that it could be gradually reduced or prevented by increasing the ELNs count.

Keywords: Gastric cancer (GC); examined lymph nodes count; stage migration; prognosis

Submitted Dec 29, 2019. Accepted for publication Jun 19, 2020.

doi: $10.21037 /$ atm-19-4727

View this article at: http://dx.doi.org/10.21037/atm-19-4727 


\section{Introduction}

Lymph node metastasis is one of the most important prognostic factors in gastric cancer (GC) patients following curative resection $(1,2)$. Since 1997, the Union for International Cancer Control (UICC) and American Joint Commission for Cancer (AJCC) have adopted categories based on the number of metastatic lymph nodes as the basis for the $\mathrm{N}$ stage of the tumor, node, metastasis (TNM) classification, in which the latest edition recommends the examination of no less than 16 regional lymph nodes for nodal metastatic status determination (3). Increasing the number of lymph nodes examined has been shown to increase the likelihood of detecting nodal metastases (4). An insufficient number of examined lymph nodes (ELNs) for diagnosis may result in underestimation of the nodal stage, a condition defined as stage migration (5).

Stage migration of lymph node metastasis is generally considered to significantly impact the patients' pathologic stage, prognostic evaluation, and strategy formulation for adjuvant therapy after surgery. Recent studies have indicated that stage migration could be gradually reduced or prevented with extension of the lymphadenectomy (the Will Rogers phenomenon) (5). Currently, as most Chinese medical centers can fulfill curative GC resection with D2 lymphadenectomy, there is theoretically little probability of stage migration. However, the scarcity of ELNs is a significant factor resulting in this phenomenon.

The eighth edition of the TNM classification designates node-negative (pN0) disease as any GC in which all ELNs are negative, regardless of the total number of ELNs (3). Several studies found that the number of ELNs was associated with the prognosis of pN0 GC patients and recommended a proper number of ELNs for pN0 GC patients (6-8). However, no studies have confirmed whether stage migration could be detected in pN0 GC patients and its possible effect on therapeutic strategies and long-term prognosis.

This multi-center collaborative analysis investigated the influence of ELNs count on stage migration and survival in pN0 GC patients after curative gastrectomy. We present the following article in accordance with the STROBE reporting checklist (available at http://dx.doi.org/10.21037/atm-19-4727).

\section{Methods}

\section{Patient selection}

Between January 2001 and December 2011, we retrospectively reviewed 7,620 GC patients undergoing surgical resection in three Chinese medical centers: 2,864 GC patients in the Department of Gastric Cancer in the Tianjin Medical University Cancer Hospital (TJMUCH), 3,043 GC patients in the Department of Surgical Oncology in The First Affiliated Hospital of China Medical University (CMUFAH) and 2,977 GC patients in the Department of Gastric and Pancreatic Surgery in the Sun Yat-sen University Cancer Center (SYSUCC), respectively. After approval from the institutional review boards of the TJMUCH, the CMUFAH and the SYSUCC, data from the cancer registries of three hospitals was obtained. The study was conducted in accordance with the Declaration of Helsinki. The study was approved by institutional ethics board of Tianjin tumor hospital (No.: bc2018037) and informed consent was taken from all the patients.

The inclusion criteria for this study included: (I) patients with histologically proven primary adenocarcinoma of the stomach; (II) patients underwent curative GC resection with a lymphadenectomy; (III) patients with pathologically negative resection margins (R0 resection). The exclusion criteria were: (I) patients with history of gastrectomy or other malignancy; (II) patients with non-curative surgical factors including distant metastasis, positive peritoneal cytology, or peritoneal dissemination; (III) patients with Siewert-I or II esophagogastric junction (EGJ) tumor; (IV) patients who died during the initial hospital stay or within 1 month after surgery; and $(\mathrm{V})$ patients who received neoadjuvant chemotherapy or radiotherapy before surgery. Based on these inclusion and exclusion criteria, we found that 71 patients had the history of gastrectomy, 46 patients had suffered from other malignant diseases, 117 patients presented with distant metastasis in the operation, 106 patients presented with the peritoneal dissemination, 503 patients were diagnosed as the Siewert-I EGJ tumor after surgery, 105 patients were identified as the R1 resection cases, 38 patients were identified as the $\mathrm{R} 2$ resection cases, 74 patients died during the first postoperative month, and 204 patients were administered with neoadjuvant treatments. Ultimately, 7,620 GC patients were included in the study (Figure 1).

\section{Patients management}

Patients underwent gastrectomy with systematic lymphadenectomy (D1+ for clinical T1N0 patients and D2 or D2+ for the others) according to the Japanese Gastric Cancer Treatment Guidelines (9). The reconstruction method was selected at the surgeon's discretion. Each medical 


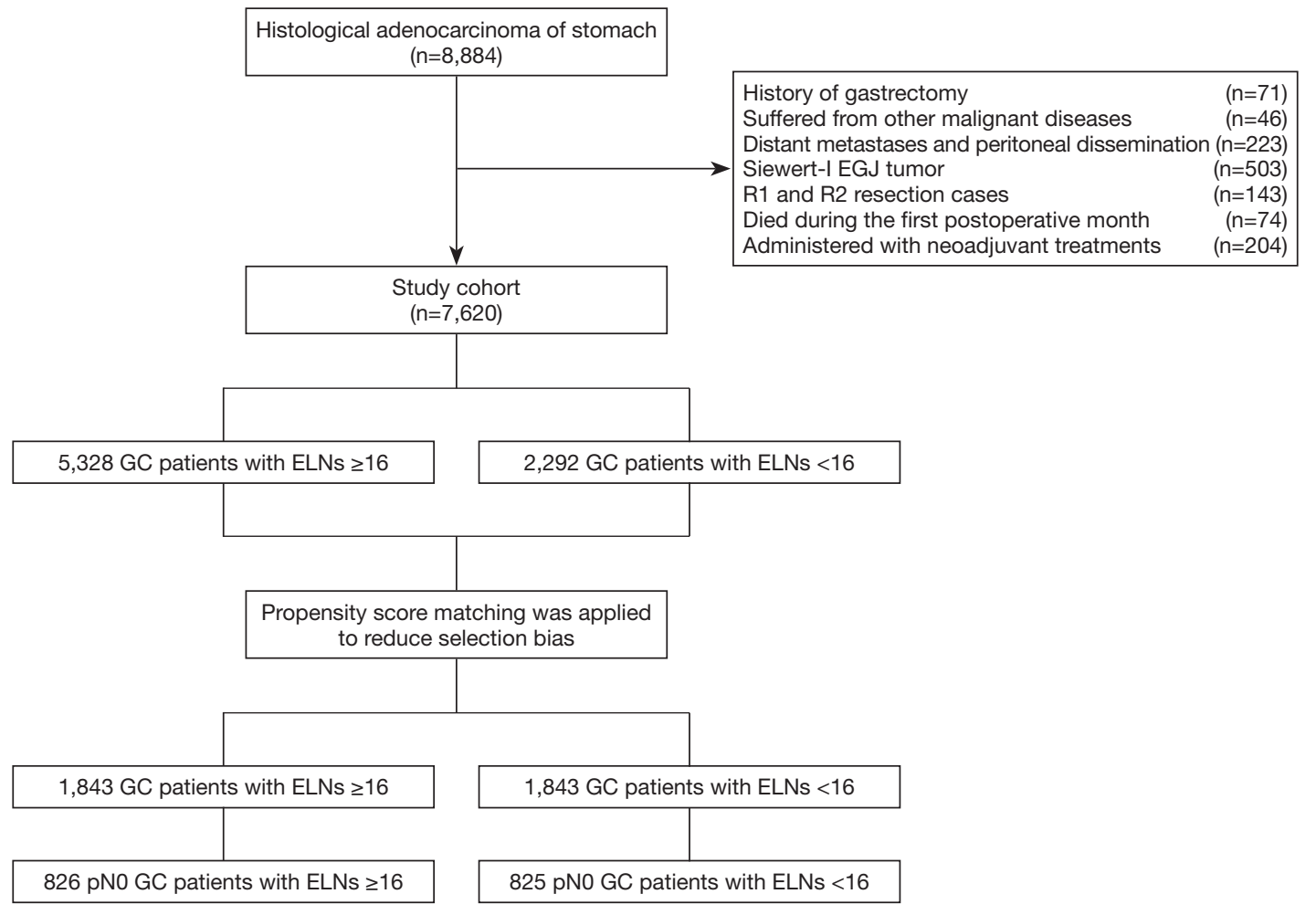

Figure 1 Patients flow diagram.

center has a surgical expert who can perform the standard gastrectomy in accordance with Japanese Gastric Cancer Treatment Guidelines. After undergoing curative surgery, all 7,620 patients were followed up every 3 or 6 months for 2 years, then every 6 months for next 3 years, and annually thereafter until death. The median follow-up time for the entire cohort was 87 (range, 2-186) months. Followup of all patients included in this study was completed in October 2015.

\section{Stage migration}

Stage migrations were mainly defined as: (I) no statistical significance of the survival differences to be detected in several subgroups of patients with different ELNs groups in the specific pTNM stages, or (II) the significant survival differences to be detected in several subgroups of patients with the same ELNs groups in the different pTNM stages.

\section{Propensity score matching (PSM)}

PSM analysis was performed. Patients with ELNs $<16$ were matched to patients with ELN $\geq 16$ based on similar estimated propensity scores to balance the covariates in the two groups and reduce selection bias (10). Propensity scores were estimated using a logistic regression model that calculated the probability of an assignment of ELNs $<16$ or $\geq 16$ based on observed baseline characteristics (11). The variables included in the model were gender, age group ( $<60$ or $\geq 60$ ), tumor location (upper-, middle-, or lower-third of the stomach or more than two-thirds of the stomach), tumor size ( $\leq 4$ or $>4 \mathrm{~cm}$ ), Lauren classification (intestinal type or diffuse type), pTNM stage, pT stage, and gastrectomy type (total or subtotal).

We used the nearest-neighbor 1:1 propensity matching without replacement method. Calculating the propensity score requires the inclusion of covariates that predict potential outcomes under each treatment arm, as well as covariates that predict treatment assignment, which are usually related $(12,13)$.

\section{Statistical analysis}

All statistical analyses were performed using IBM SPSS 
Statistics (Version 24.0; IBM Corp, New York, USA). $\chi^{2}$ and $t$-tests were used for categorical and continuous variables, respectively. The probabilities of overall survival (OS) were calculated by the Kaplan-Meier method and the differences in OS probabilities between groups were examined by logrank tests. Stage-stratified survival analyses were conducted according to the 8th Edition of the AJCC pathological TNM system. Multivariate Cox proportional hazards regression was used to evaluate the independent prognostic risk factors of OS and to calculate hazard ratios (HRs) with $95 \%$ confidence intervals (CIs). In all analyses, two-tailed $\mathrm{P}<0.05$ was considered statistically significant.

\section{Results}

\section{Baseline data before and after matching}

Of the 7,620 GC patients, 2,292 (30.0\%) had <16 ELNs and 5,328 (70.0\%) had $\geq 16$ ELNs. After PSM, there were 1,843 patients in each group. The baseline data of the whole cohort before and after PSM are summarized in Table 1. Before matching, differences were observed in terms of age $(\mathrm{P}<0.001)$, gender $(\mathrm{P}<0.001)$, tumor location $(\mathrm{P}<0.001)$, tumor size $(\mathrm{P}=0.022)$, Lauren classification $(\mathrm{P}=0.001)$, pTNM stage $(\mathrm{P}<0.001)$, pT stage $(\mathrm{P}<0.001)$, pN stage $(\mathrm{P}<0.001)$ and gastrectomy type $(\mathrm{P}<0.001)$; after matching, the results were similar between the two groups $(\mathrm{P}>0.05$; Table 1$)$.

We further analyzed the node-negative GC patients. Before PSM, there were 1,031 pN0 GC patients with ELNs $<16$ and 1,762 pN0 GC patients with ELNs $\geq 16$. After PSM, there were 825 patients with ELNs $<16$ and 826 patients with ELNs $\geq 16$. The characteristics of the nodenegative GC patients before and after PSM are summarized in Table 2. Of the $2793 \mathrm{pN} 0 \mathrm{GC}$ patients, differences were observed in terms of age $(\mathrm{P}<0.001)$, gender $(\mathrm{P}=0.001)$, tumor location $(\mathrm{P}<0.001)$, tumor size $(\mathrm{P}=0.022)$, pTNM stage $(\mathrm{P}<0.001)$, pT stage $(\mathrm{P}<0.001)$, and gastrectomy type $(\mathrm{P}=0.001)$ before matching. After matching, the backgrounds of pN0 GC patients in the ELNs $<16(n=825)$ and ELNs $\geq 16$ ( $n=826)$ groups were well-balanced.

The mean $( \pm$ SD) number of pathologically proven ELNs for the matched cohort of 1,651 node-negative GC patients was $18.5 \pm 11.5$ (range: $1-87$ ), with $9.9 \pm 3.8$ in the ELNs $<16$ group and $27.0 \pm 10.2$ in the ELNs $\geq 16$ group.

\section{Survival factor analysis for the matched pNO GC patients}

The ELNs $\geq 16$ group had a longer OS (HR 0.644; 95\% CI,
0.516-0.804, $\mathrm{P}=0.001$ ) (Figure 2) than that in the ELNs $<16$ group. The 5-year survival rate (5-YSR) in the two groups were $82.6 \%$ and $76.3 \%$, respectively. Univariate analysis revealed that the following seven clinicopathological characteristics were significantly associated with OS in the matched pN0 GC patients: age at surgery, tumor location, tumor size, Lauren classification, type of gastrectomy, pTNM stage, and ELNs count (Table 3). These characteristics were then included in a multivariate Cox proportional hazard model (forward stepwise procedure) to adjust for the effects of covariates. Multivariate analysis indicated that ELNs count (HR, 0.644; $\mathrm{P}<0.001)$, age at surgery, tumor location, tumor size, Lauren classification, and type of gastrectomy were independent predictors of OS in pN0 GC patients (Table 3).

\section{Subgroup analyses for the matched pNO GC patients}

We performed subgroup analyses of node-negative GC patients to further investigate the clinical significance of ELNs. Tables 4,5 and Figure 3 show the subgroup survival analyses for pTNM status between the ELNs $<16$ and ELNs $\geq 16$ groups. Based on the aforementioned definition, stage migrations were observed in several subgroups of $\mathrm{pN} 0$ GC patients with specific pTNM stages: (I) pT2N0M0 $($ ELNs < 16) vs. pT3N0M0 (ELNs $\geq 16)(\mathrm{P}=0.371$, Figure 3B); (II) pT2N0M0 (ELNs <16) vs. pT4aN0M0 (ELNs $\geq 16)(\mathrm{P}=0.100$, Figure $3 D)$; (III) pT3N0M0 (ELNs <16) vs. pT4aN0M0 (ELNs $\geq 16)(\mathrm{P}=0.859$, Figure $3 F)$; (IV) pT3N0M0 ( ELNs <16) vs. pT4bN0M0 (ELNs $\geq 16)$ $(\mathrm{P}=0.397$, Figure $3 H)$; and $(\mathrm{V}) \mathrm{pT} 4 \mathrm{aN0M0}(\mathrm{ELNs}<16) v s$. pT4bN0M0 (ELNs $\geq 16)(\mathrm{P}=0.831$, Figure 37).

Stage migration was detected between pT2N0M0 (ELNs $<16)$ and pT3N0M0 (ELNs $\geq 16)(\mathrm{P}=0.371$, Figure $3 B)$. Since $\mathrm{p} T$ stage is unalterable, the causes of stage migration were mostly attributed to the alteration of $\mathrm{pN}$ stage. We hypothesized that an increasing number of ELNs may transform stage pT2N0M0 GC patients into stage pT2N1M0. We used the matched all-stage GC patients to test this hypothesis. Figure $3 C$ shows that there was no difference in stage pT2N0M0 (ELNs <16) and pT2N1M0 $(E L N s \geq 16)(\mathrm{P}=0.214)$ when using the matched all-stage GC patients for analysis. Thus, the hypothesis was proven: stage pT2N0M0 GC patients may be classified as stage pT2N1M0 with an increased ELN count.

Similarly, we repeated this verification process between: (I) pT2N0M0 (ELNs <16) vs. pT4aN0M0 (ELNs $\geq 16$ ), (II) pT3N0M0 (ELNs <16) vs. pT4aN0M0 (ELNs $\geq 16$ ), (III) 
Table 1 Baseline data before and after propensity score matching of the whole cohort

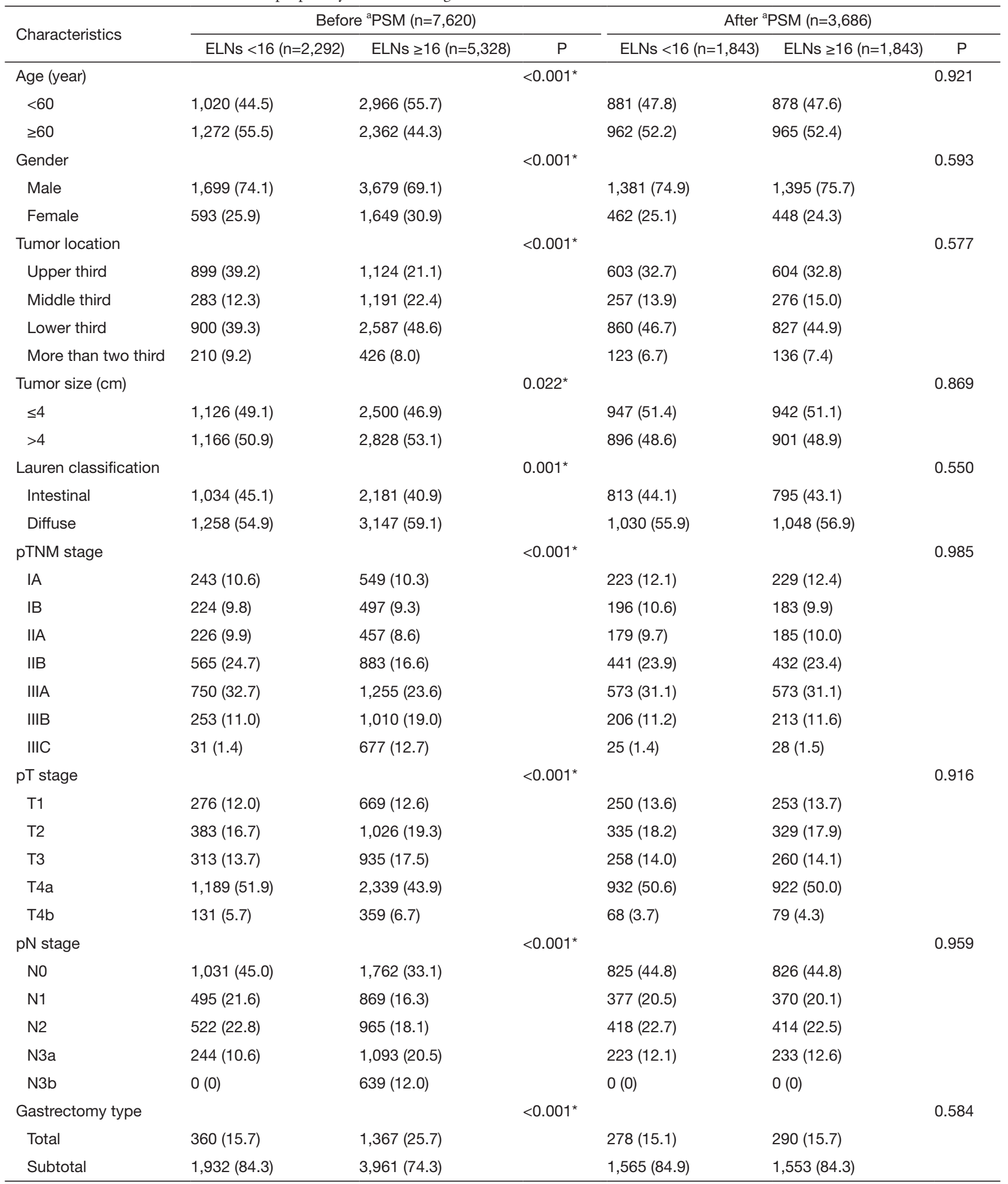

Data presented as No. (\%) unless otherwise noted. *, $\mathrm{P}<0.05$; ${ }^{\text {, }}$ matched parameters - gender, age group, tumor location, tumor size, Lauren classification, pTNM stage, pT stage, and gastrectomy type. ELNs, examined lymph nodes; PSM, propensity score matching. 
Table 2 Baseline data before and after propensity score matching of pN0 GC patients

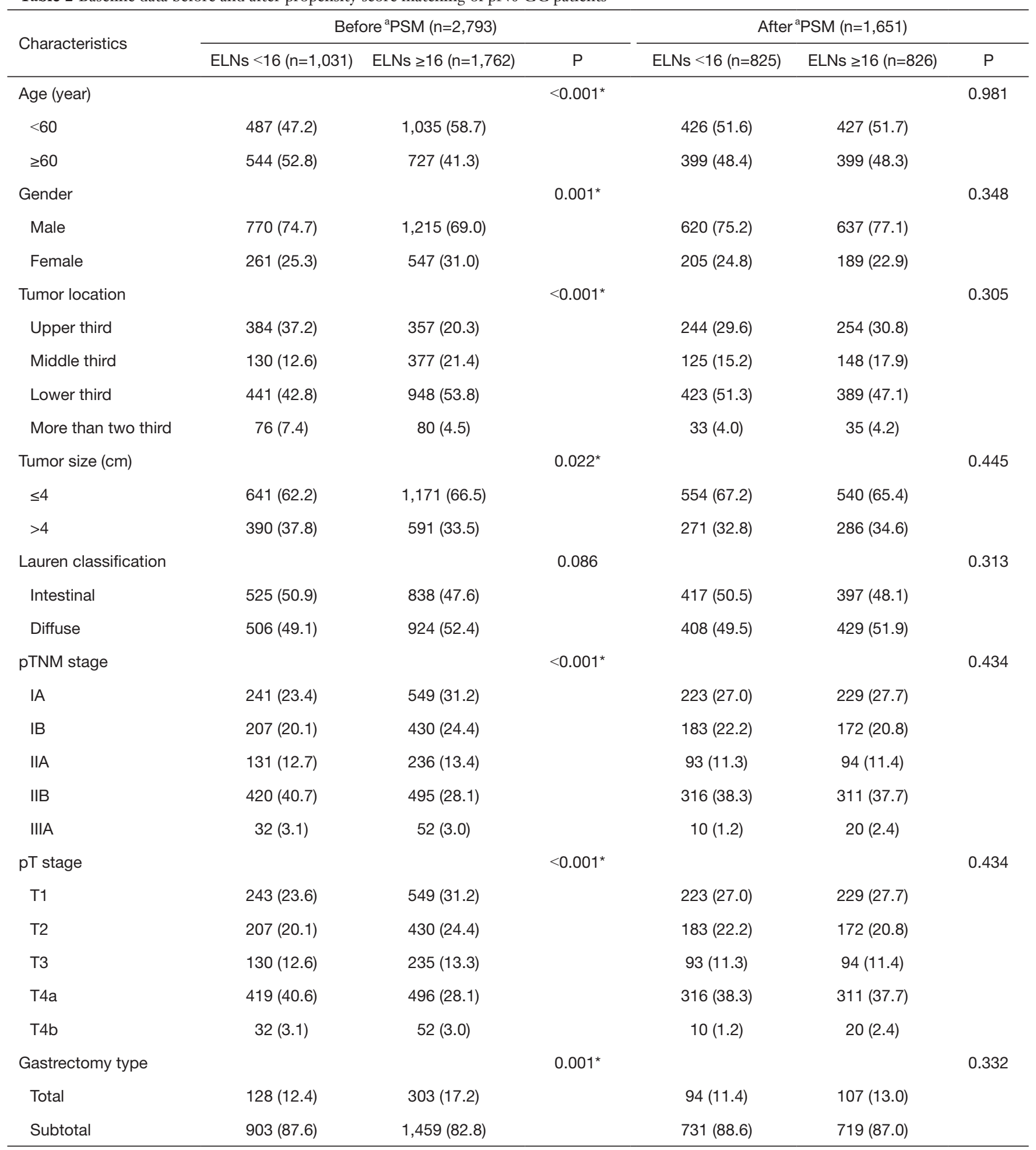

Data presented as No. (\%) unless otherwise noted. * $\mathrm{P}<0.05$; ${ }^{\text {a }}$, matched parameters-gender, age group, tumor location, tumor size, Lauren classification, pTNM stage, pT stage, and gastrectomy type. ELNs, examined lymph nodes; PSM, propensity score matching. 
pT3N0M0 (ELNs <16) vs. pT4bN0M0 (ELNs $\geq 16$ ), and (IV) pT4aN0M0 (ELNs <16) vs. pT4bN0M0 (ELNs $\geq 16$ ). The hypotheses were disproven in: (I) pT2N0M0 (ELNs <16) vs. pT2N2M0 (ELNs $\geq 16)(\mathrm{P}=0.004$, Figure $3 E)$ and (II) pT3N0M0 (ELNs <16) vs. pT3N2M0 (ELNs $\geq 16)(\mathrm{P}<0.001$, Figure 31$)$. The hypotheses were proven in: (I) pT3N0M0 (ELNs <16) vs. pT3N1M0 $($ ELNs $\geq 16)$ $(\mathrm{P}=0.079$, Figure $3 G)$ and (II) pT4aN0M0 $\left(\mathrm{ELNs}_{<}<16\right)$ vs. pT4aN1M0 and pT4aN2M0 (ELNs $\geq 16$ ) (Figure $3 K$ ). Stage

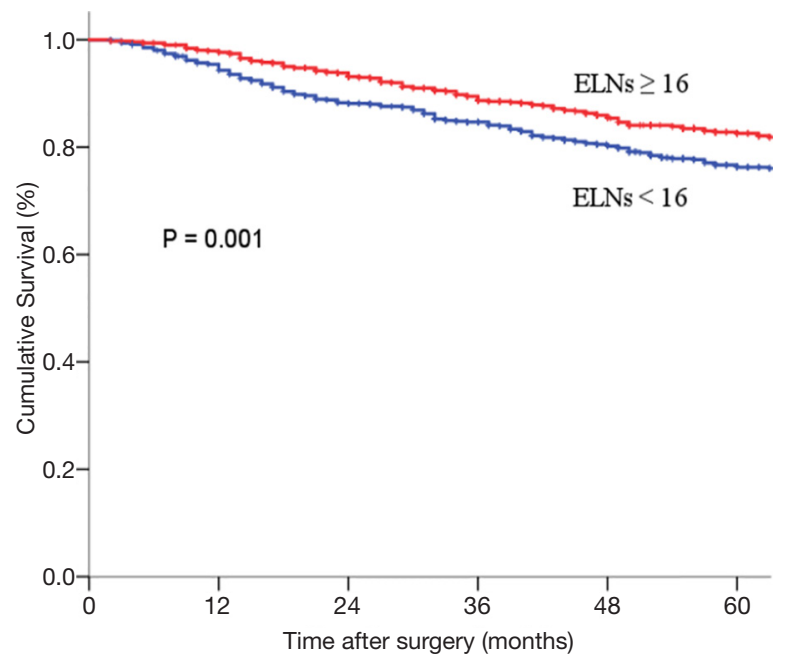

No. at risk

$\begin{array}{lllllll}\text { ELNs }<16 & 825 & 759 & 663 & 585 & 474 & 369\end{array}$

Figure 2 Survival curves for the matched pN0 GC patients with ELNs $<16(n=825)$ or ELNs $\geq 16(n=826)$. ELNs, examined lymph nodes.
pT4aN0M0 may migrate to pT4aN1M0 $(\mathrm{P}=0.921)$ rather than to pT4aN2M0 $(\mathrm{P}<0.001)$.

\section{Discussion}

Although several new staging methods have been proposed to evaluate the prognosis of $\mathrm{GC}$, the TNM staging system based on the count of positive lymph nodes is still widely used as the number of positive lymph nodes well reflects the prognosis (14-16). Recently, the AJCC eighth staging system made several modifications mainly based on recommendations from the International Gastric Cancer Association Staging Project (3). Although the optimal number of ELNs remains controversial, the AJCC eighth GC staging system traditionally recommends the examination of at least 16 lymph nodes. Notably, only about $37 \%(1,032 / 2,793)$ of the node-negative GC patients in our study had the AJCC-recommended minimum of 16 ELNs, which meant that more than half of the node-negative GC patients were inappropriately evaluated according to the AJCC eighth staging system. GC patients might be staged incorrectly because of an insufficient number of ELNs (17). Our previous study showed that patients with ELNs $\geq 16$ had a significantly higher median OS than those with ELNs $<16$ after curative surgery because of the underestimation of the $\mathrm{N}$ stage of patients with ELNs $<16$ (18). We also demonstrated that an insufficient ELNs count may be a potential risk factor for the postoperative recurrence even in node-negative GC patients (19).

The number of ELNs refers to the total number of lymph nodes obtained from radical gastrectomy specimens

Table 3 Univariable and multivariable analysis of matched node-negative GC patients

\begin{tabular}{|c|c|c|c|c|c|c|}
\hline Characteristics & \multicolumn{3}{|c|}{ Univariable analysis } & \multicolumn{3}{|c|}{ Multivariable analysis } \\
\hline Age & 1.499 & $1.196-1.878$ & $<0.001^{*}$ & 1.522 & $1.215-1.906$ & $<0.001^{\star}$ \\
\hline Gender & 0.782 & $0.595-1.027$ & 0.077 & & & \\
\hline Tumor location & 1.119 & $1.002-1.250$ & $0.046^{\star}$ & 1.121 & $1.004-1.253$ & $0.043^{*}$ \\
\hline Lauren classification & 1.578 & $1.245-2.001$ & $<0.001^{*}$ & 1.539 & $1.216-1.949$ & $<0.001^{*}$ \\
\hline pTNM stage & 1.720 & 1.539-1.922 & $<0.001^{*}$ & 1.719 & $1.538-1.922$ & $<0.001^{\star}$ \\
\hline Gastrectomy type & 0.587 & $0.444-0.775$ & $<0.001^{*}$ & 0.577 & $0.437-0.762$ & $<0.001^{*}$ \\
\hline ELNs & 0.642 & $0.515-0.801$ & $<0.001^{*}$ & 0.644 & $0.516-0.804$ & $<0.001^{*}$ \\
\hline
\end{tabular}

${ }^{*}, \mathrm{P}<0.05$. HR, hazard ratio; $\mathrm{Cl}$, confidence interval. 
Table 4 Subgroup survival analyses for pTNM status between ELNs $<16$ group and ELNs $\geq 16$ group for matched pN0 GC patients (part A)

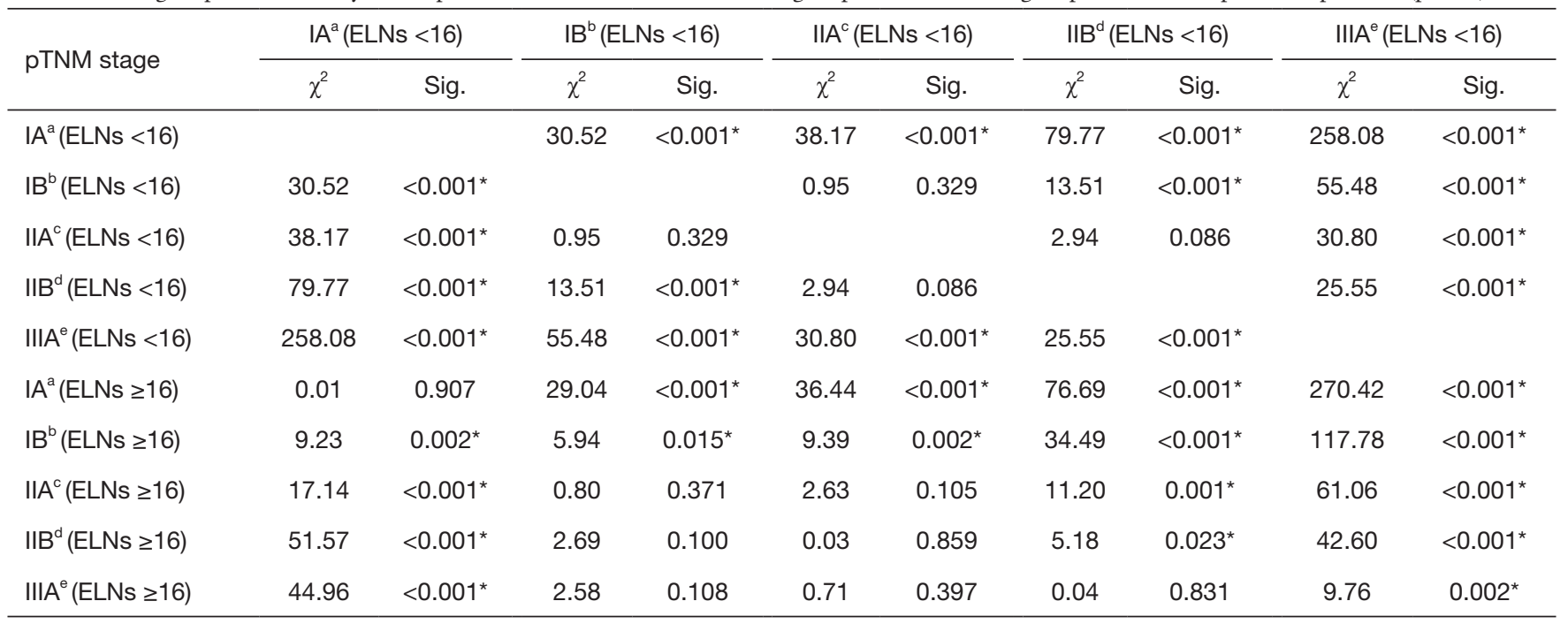

a, pT1NOMO; ${ }^{\text {, }}$ pT2NOMO; c, pT3NOM0; ${ }^{\text {, }}$, pT4aNOMO; ${ }^{\text {, }}$ pT4bNOMO; *, P<0.05. ELNs, examined lymph node counts; Sig, significance.

Table 5 Subgroup survival analyses for pTNM status between ELNs $<16$ group and ELNs $\geq 16$ group for matched pN0 GC patients (part B)

\begin{tabular}{|c|c|c|c|c|c|c|c|c|c|c|}
\hline \multirow{2}{*}{ pTNM stage } & \multicolumn{2}{|c|}{$\mathrm{IA}^{\mathrm{a}}(\mathrm{ELNs} \geq 16)$} & \multicolumn{2}{|c|}{$\mathrm{IB}^{\mathrm{b}}(\mathrm{ELNs} \geq 16)$} & \multicolumn{2}{|c|}{$\| A^{c}(E L N s \geq 16)$} & \multicolumn{2}{|c|}{ IIB $^{\mathrm{d}}(\mathrm{ELNs} \geq 16)$} & \multicolumn{2}{|c|}{$\mathrm{IIIA}^{\mathrm{e}}(\mathrm{ELNs} \geq 16)$} \\
\hline & $\chi^{2}$ & Sig. & $\chi^{2}$ & Sig. & $\chi^{2}$ & Sig. & $\chi^{2}$ & Sig. & $\chi^{2}$ & Sig. \\
\hline $\mathrm{IA}^{\mathrm{a}}(\mathrm{ELNs}<16)$ & 0.014 & 0.907 & 9.23 & $0.002^{*}$ & 17.14 & $<0.001^{*}$ & 51.57 & $<0.001^{*}$ & 44.96 & $<0.001^{*}$ \\
\hline $\mathrm{IB}^{\mathrm{b}}(\mathrm{ELNs}<16)$ & 29.04 & $<0.001^{*}$ & 5.94 & $0.015^{\star}$ & 0.80 & 0.371 & 2.69 & 0.100 & 2.58 & 0.108 \\
\hline$\| A^{c}(E L N s<16)$ & 36.44 & $<0.001^{*}$ & 9.39 & $0.002^{*}$ & 2.63 & 0.105 & 0.03 & 0.859 & 0.71 & 0.397 \\
\hline IIIA ${ }^{e}(E L N s<16)$ & 270.42 & $<0.001^{\star}$ & 117.78 & $<0.001^{\star}$ & 61.06 & $<0.001^{\star}$ & 42.60 & $<0.001^{\star}$ & 9.76 & $0.002^{*}$ \\
\hline$I^{a}(E L N s \geq 16)$ & & & 8.71 & $0.003^{\star}$ & 16.84 & $<0.001^{*}$ & 50.61 & $<0.001^{\star}$ & 43.28 & $<0.001^{\star}$ \\
\hline $\mathrm{IB}^{\mathrm{b}}(\mathrm{ELNs} \geq 16)$ & 8.71 & $0.003^{\star}$ & & & 1.28 & 0.256 & 16.83 & $<0.001^{\star}$ & 10.92 & $0.001^{*}$ \\
\hline$\| A^{c}(E L N s \geq 16)$ & 16.84 & $<0.001^{\star}$ & 1.28 & 0.256 & & & 4.01 & $0.045^{\star}$ & 4.31 & $0.038^{*}$ \\
\hline
\end{tabular}

a , pT1NOMO; ${ }^{\text {b }}$ pT2NOMO; ${ }^{\text {, }}$ pT3NOM0; ${ }^{\text {d }}$, pT4aNOMO; ${ }^{\text {e }}$, pT4bNOMO; * P<0.05. ELNs, examined lymph node counts; Sig, significance.

during the pathological examination, rather than the total number of lymph nodes dissected (4). Theoretically, since the number of lymph nodes in all soft tissues (especially the small and occult ones) cannot be determined, the number of lymph nodes should not exceed the number of lymph nodes dissected. To obtain lymph nodes for examination, special personnel are required to sort each station of lymph nodes in the perigastric region from the surgical specimens of $\mathrm{GC}$, making detailed records before sending the samples for examination. Unfortunately, many medical centers in China and many foreign countries do not pay attention to this step, resulting in a relatively low number of lymph nodes for final postoperative pathological examination. In addition, since many pathologists are not aware of the anatomical location of the perigastric gastric lymph nodes, it is more difficult to sort out enough lymph nodes to detect metastasis.

Generally, stage migration of lymph node metastasis is considered to significantly impact patients' pathological stage, prognostic evaluation, and strategy formulation for adjuvant therapy after surgery. Stage migration of lymph 
A

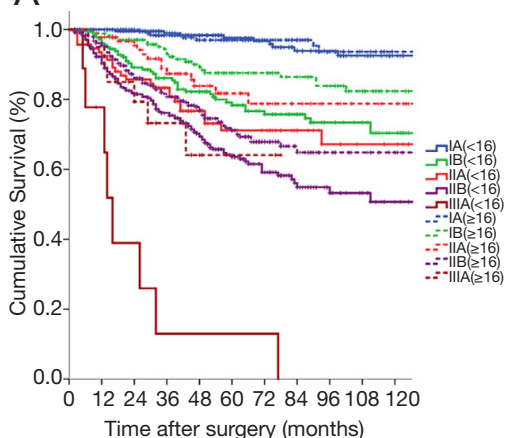

D

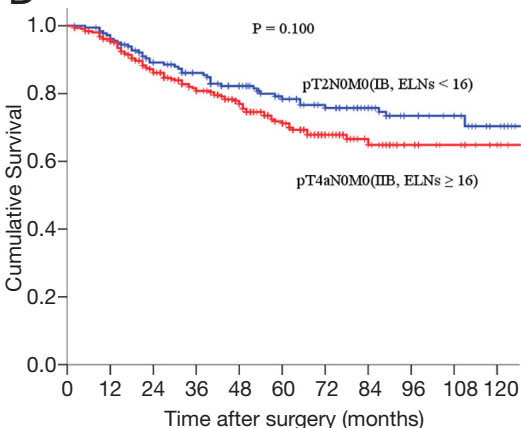

G

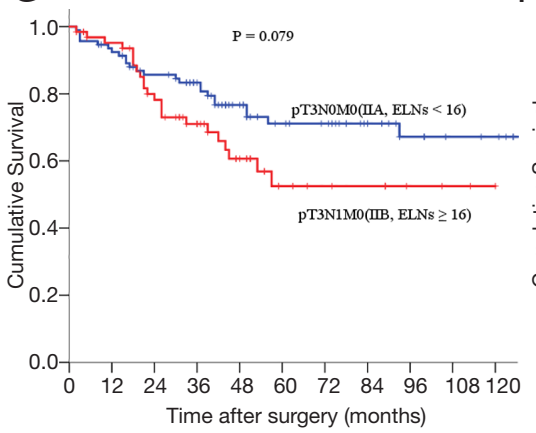

J

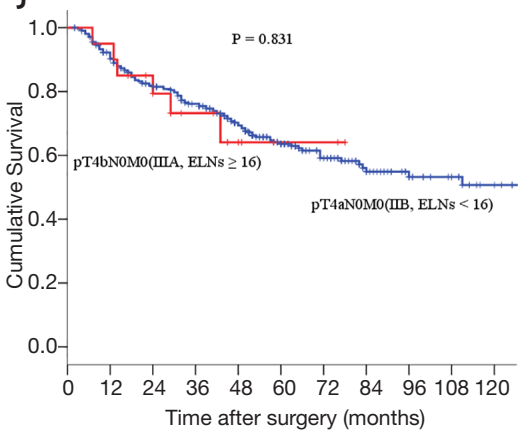

B

E

K
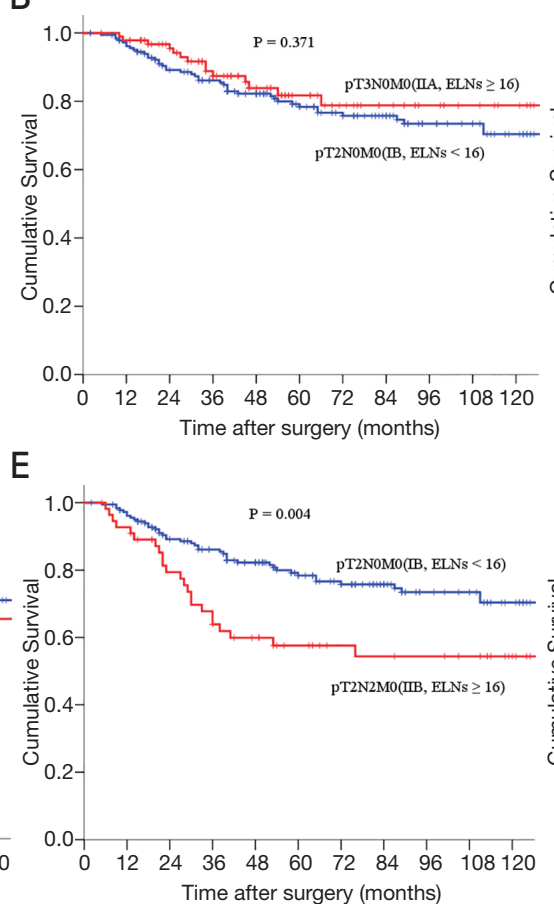

$\mathrm{H}$
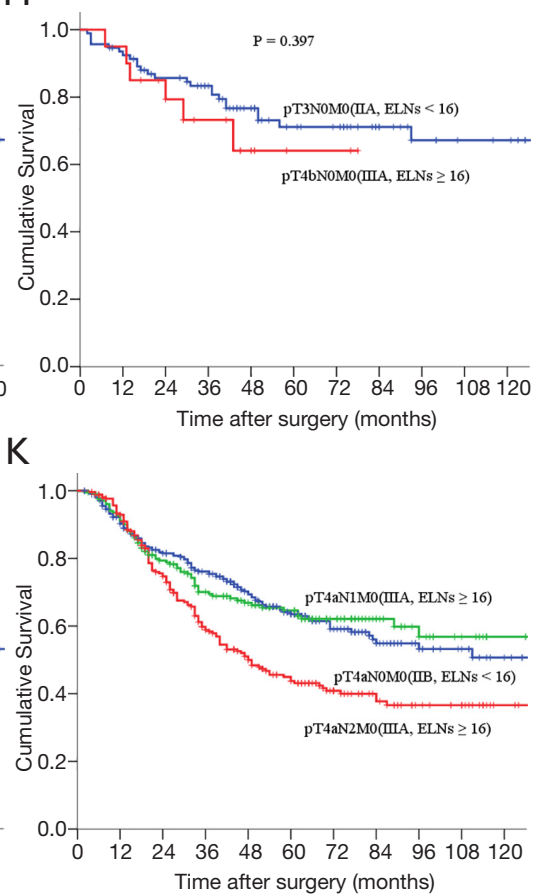

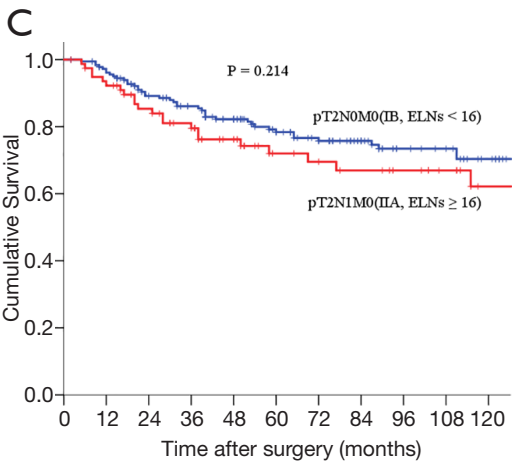

F
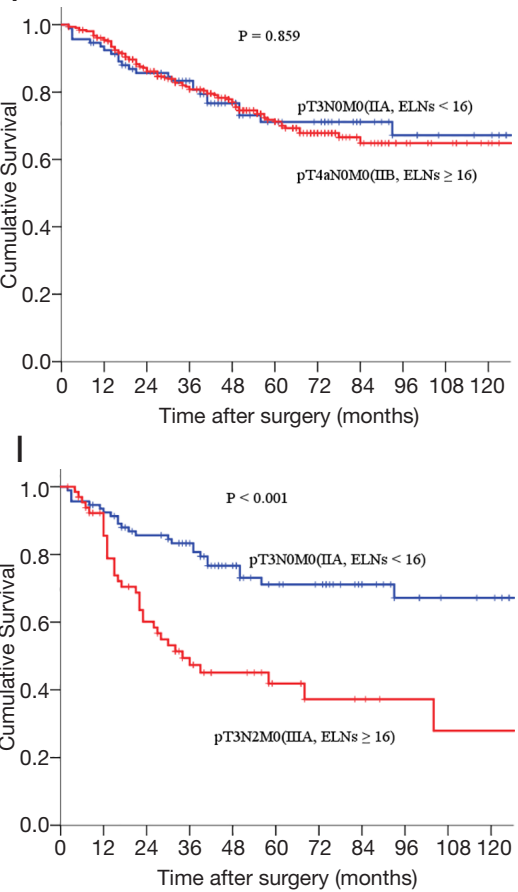

Figure 3 Subgroup survival analyses for the matched pN0 GC patients. (A) Survival analysis for pTNM stage between ELNs $<16$ group and ELNs $\geq 16$ group; (B) pT2N0M0 (IB, ELNs <16) vs. pT3N0M0 ( IIA, ELNs $\geq 16$ ) (P=0.371); (C) pT2N0M0 (IB, ELNs <16) vs. pT2N1M0 (IIA, ELNs $\geq 16$ ) (P=0.214); (D) pT2N0M0 (IB, ELNs <16) vs. pT4aN0M0 (IIB, ELNs $\geq 16)(\mathrm{P}=0.100)$; (E) pT2N0M0 (IB, ELNs $<16) v s$. pT2N2M0 (IIB, ELNs $\geq 16$ ) (P=0.004); (F) pT3N0M0 (IIA, ELNs <16) vs. pT4aN0M0 (IIB, ELNs $\geq 16$ ) ( $\mathrm{P}=0.859)$; (G) pT3N0M0 (IIA, ELNs <16) vs. pT3N1M0 (IIB, ELNs $\geq 16)(\mathrm{P}=0.079)$; (H) pT3N0M0 (IIA, ELNs <16) vs. pT4bN0M0 (IIIA, ELNs $\geq 16)(\mathrm{P}=0.397) ;(\mathrm{I})$ pT3N0M0 (IIA, ELNs <16) vs. pT3N2M0 (IIIA, ELNs $\geq 16$ ) (P<0.001); (J) pT4aN0M0 (IIB, ELNs <16) vs. pT4bN0M0 (IIIA, ELNs $\geq 16$ ) $(\mathrm{P}=0.831)$; (K) pT4aN0M0 (IIB, ELNs <16) vs. pT4aN1M0 and pT4aN2M0 (IIIA, ELNs $\geq 16$ ). ELNs, examined lymph nodes. 
node metastasis may be induced by individual differences in patients, disease stage, special biological behaviors of the tumor, extent of lymphadenectomy, procedures of lymph node examination, etc., which can hardly give rise to the accurate staging of lymph node metastasis from GC in clinical settings. The specification procedures for lymph node examination following radical lymphadenectomy for GC are not only the necessary method to obtain comprehensive information on lymph node metastasis from patients but are also essential evidence for high-quality curative gastrectomy for patients, and which may provide an exhaustive database for further precise GC diagnosis and treatment (4).

Stage migration of lymph node metastasis was initially reported in GC as a directly proportional relationship between the number of metastatic lymph nodes and the extent of lymphadenectomy; and with the expansion of lymphadenectomy, the stage migration of lymph nodes metastasis can be gradually reduced or avoided (the so-called Will Rogers phenomenon) (5). Currently, most Chinese medical centers can fulfill curative GC resection with D2 lymphadenectomy; theoretically, there is little probability of stage migration. However, the scarcity of ELNs is a significant factor resulting in this phenomenon. Therefore, in recent years many scholars have proposed a variety of new evaluation indicators to reduce the stage migration of lymph node metastasis, including the lymph node metastasis rate, $\log$ odds of positive lymph nodes, and the ratio of negative and positive lymph nodes (20-22). However, while the above index can partially make up the deficiency of lymph nodes stage migration for evaluating the prognosis of patients with GC after radical gastrectomy in a single medical center, we should remember that these indicators are just a mathematical method for changing a constant to a ratio or logarithm and its role in reducing stage migration is limited, making it difficult to achieve the same effect in a wide geographical range or in multiple centers (23).

The clinical data of patients with radical gastrectomy in our center in the past ten years suggests that the number of metastatic lymph nodes in patients after D2 lymphadenectomy increased with the addition of the number of ELNs for positive correlation (18). As early as 2005, Smith et al. found that every 10 additional ELNs can improve the prognosis of GC patients to some extent in an analysis of GC patient's data in the US Surveillance, Epidemiology, and End Results (SEER) database (24). The reason may be closely related to stage migration and local disease control. In 2009, according to the sixth edition of the TNM staging system of GC, our study also showed that patients with ELNs $\geq 15$ experienced significantly better total survival, disease-free survival, and survival after recurrence than those in patients with ELNs <15 (25). In analysis of D2 lymphadenectomy, which improves the prognosis of some patients with GC, we also found that an increased ELNs count was an independent prognostic factor of GC patients with perigastric lymph nodes metastasis (greater and lesser curvature) (26). When the 7 th edition of GC TNM staging system was issued, the recommended number of ELNs was not less than 16 because stage $\mathrm{pN} 3 \mathrm{~b}$ patients required at least 16 metastatic lymph nodes to be confirmed by pathology and there were reports that patients with ELNs $\geq 16$ had significantly higher survival rates than those in patients with ELNs $<16$ (18).

It's feasible to correct for stage migration of lymph node metastases by increasing the number of ELNs. The surgical treatment of GC in Japan and South Korea has always been significantly ahead of that in China, partly due to the fact that Japanese and Koreans pay more attention to early screening of GC, resulting in a much higher proportion of early GC than that in China. However, the postoperative survival rates of patients with advanced GC in Japan and South Korea are also significantly higher than that of developed medical centers in China. The popularity of D2 lymphadenectomy in most medical centers in China does not seem to have the same effect in Japan and South Korea; thus, it is not entirely due to surgical skills. Recently, Sano et al. found that the proportion of $\mathrm{pN} 3 \mathrm{~b}$ GC patients was almost two times higher in some East Asian countries except for Japanese and South Korea (including 979 patients in China) with a relatively low number of lymph nodes (24.8 per case) than that in Japan and South Korea (3). This finding shows that the number of metastasis lymph nodes in GC patients in China may increase with an increased ELNs count. Our retrospective analysis of the clinical and pathological data of 7,620 GC patients undergoing radical gastrectomy revealed that node-positive patients with ELNs $\geq 30$ had the highest survival rate in the same subgroup of patients with $\mathrm{pN}$ stage, which is also the prognosis-related stage migration caused by an insufficient number of ELNs we are currently facing (4).

In the present study, we initially explored the setting in which the ELNs strongly influenced the prognosis and stage migration of node-negative patients. For this purpose, we employed a multicenter collaborative database to select patients with pN0 GC who underwent a total gastrectomy. The latest edition staging system designates node- 
negative disease as any GC in which all ELNs are negative, regardless of the total number of ELNs. Node-negative GC is an easily overlooked disease with a relatively favorable prognosis and the treatment is quite different from that of advanced GC. If a node-positive patient is staged incorrectly as node-negative because of an insufficient number of ELNs, the therapeutic strategies and long-term prognosis would be greatly affected. Thus, there is an urgent need to recognize the significance of ELNs.

We employed PSM to minimize selection bias before conducting multivariable analysis and found that the number of ELNs was an independent prognostic factor in node-negative GC patients (Table 2), a finding consistent with those of previous studies $(27,28)$. The association between the number of ELNs and OS was mostly attributed to stage migration. Node-negative GC patients with few ELNs may not truly node-negative but rather indicate the presence of understaging. Increased lymph node removal will also reduce the chance of inappropriate understaging. Subgroup analyses revealed stage migration between several subgroups: (I) pT2N0M0 (ELNs <16) vs. pT3N0M0 (ELNs $\geq 16)(\mathrm{P}=0.371$, Figure $3 B)$; (II) pT2N0M0 (ELNs $<16)$ vs. pT4aN0M0 (ELNs $\geq 16)(\mathrm{P}=0.100$, Figure $3 D)$; (III) pT3N0M0 (ELNs <16) vs. pT4aN0M0 (ELNs $\geq 16$ ) $(\mathrm{P}=0.859$, Figure $3 F)$; (IV) p T3N0M0 (ELNs <16) vs. pT4bN0M0 (ELNs $\geq 16)(\mathrm{P}=0.397$, Figure $3 H)$; and (V) pT4aN0M0 (ELNs <16) vs. stage pT4bN0M0 (ELNs $\geq 16)(\mathrm{P}=0.831$, Figure 37). We then made hypotheses and used the all-stage matched GC patients to test them. Some hypotheses were proven, indicating the presence of certain stage migrations and that increasing the ELNs count could induce the alteration of pTNM staging in node-negative GC patients.

The present study has several limitations. The main limitation of the work we present here is its retrospective population-study design. The great disadvantage of population studies is their inherent variability. This variability is derived both from differences in the study centers ( 3 centers in our case) and in the possible variation in the surgical techniques used, surgeons' specializations and preferences. Also, all patients are come from Chinese population in this study, which perhaps result in little bias of detection results comparing to the other race.

\section{Conclusions}

Stage migration could be detected in pN0 GC patients, especially in cases with an insufficient number of ELNs.
However, increasing the number of ELNs could avoid the stage migration phenomenon. We recommend at least 16 ELNs for correct staging, even in patients with nodenegative GC.

\section{Acknowledgments}

Funding: This study was supported in part by grants from the Programs of National Natural Science Foundation of China (No. 81572372, No. 81172080, No. 81201773, No. 81572372), National Key Research and Development Program (MOST-2016YFC1303202), National Precision Medicine Research Program (2017YFC0908304) and the National Key Clinical Specialist Construction Programs of China [2013-544].

\section{Footnote}

Reporting Checklist: The authors have completed the STROBE reporting checklist. Available at http://dx.doi. org/10.21037/atm-19-4727

Data Sharing Statement: Available at http://dx.doi. org/10.21037/atm-19-4727

Conflicts of Interest: All authors have completed the ICMJE uniform disclosure form (available at http://dx.doi. org/10.21037/atm-19-4727). The authors have no conflicts of interest to declare.

Ethical Statement: The authors are accountable for all aspects of the work in ensuring that questions related to the accuracy or integrity of any part of the work are appropriately investigated and resolved. The study was conducted in accordance with the Declaration of Helsinki (as revised in 2013). The study was approved by institutional ethics board of Tianjin tumor hospital (No.: bc2018037) and informed consent was taken from all the patients.

Open Access Statement: This is an Open Access article distributed in accordance with the Creative Commons Attribution-NonCommercial-NoDerivs 4.0 International License (CC BY-NC-ND 4.0), which permits the noncommercial replication and distribution of the article with the strict proviso that no changes or edits are made and the original work is properly cited (including links to both the formal publication through the relevant DOI and the license). See: https://creativecommons.org/licenses/by-nc-nd/4.0/. 


\section{References}

1. Sasako M, Saka M, Fukagawa T, et al. Surgical treatment of advanced gastric cancer: Japanese perspective. Dig Surg 2007;24:101-7.

2. Hartgrink HH, Jansen EP, van Grieken NC, et al. Gastric cancer. Lancet 2009;374:477-90.

3. Sano T, Coit DG, Kim HH, et al. Proposal of a new stage grouping of gastric cancer for TNM classification: International Gastric Cancer Association staging project. Gastric Cancer 2017;20:217-25.

4. Deng J, Liu J, Wang W, et al. Validation of clinical significance of examined lymph node count for accurate prognostic evaluation of gastric cancer for the eighth edition of the American Joint Committee on Cancer (AJCC) TNM staging system. Chin J Cancer Res 2018;30:477-91.

5. de Manzoni G, Verlato G, Roviello F, et al. The new TNM classification of lymph node metastasis minimises stage migration problems in gastric cancer patients. Br J Cancer 2002;87:171-4.

6. Xu D, Huang Y, Geng Q, et al. Effect of lymph node number on survival of patients with lymph node-negative gastric cancer according to the 7 th edition UICC TNM system. PloS One 2012; 7:e38681.

7. Baiocchi GL, Tiberio GA, Minicozzi AM, et al. A multicentric Western analysis of prognostic factors in advanced, node-negative gastric cancer patients. Ann Surg 2010;252:70-3.

8. Jiao XG, Deng JY, Zhang RP, et al. Prognostic value of number of examined lymph nodes in patients with node-negative gastric cancer. World J Gastroenterol 2014;20:3640-8.

9. Nakajima T. Gastric cancer treatment guidelines in Japan. Gastric Cancer 2002;5:1-5.

10. D'Agostino RB Jr. Propensity score methods for bias reduction in the comparison of a treatment to a nonrandomized control group. Stat Med 1998;17:2265-81.

11. Austin PC. An introduction to propensity score methods for reducing the effects of confounding in observational studies. Multivariate Behav Res 2011;46:399-424.

12. McMurry TL, Hu Y, Blackstone EH, et al. Propensity scores: methods, considerations, and applications in the journal of thoracic and cardiovascular surgery. J Thorac Cardiovasc Surg 2015;150:14-9.

13. Elze MC, Gregson J, Baber U, et al. Comparison of propensity score methods and covariate adjustment: evaluation in 4 cardiovascular studies. J Am Coll Cardiol
2017;69:345-57.

14. Deng J, Sun D, Pan Y, et al. Ratio between Negative and Positive Lymph Nodes Is Suitable for Evaluation the Prognosis of Gastric Cancer Patients with Positive Node Metastasis. PLoS One 2012; 7:e43925.

15. Deng J, Liang H, Sun D, et al. Suitability of 7th UICC N stage for predicting the overall survival of gastric cancer patients after curative resection in China. Ann Surg Oncol 2010;17:1259-66.

16. Deng J, Liang H, Wang D, et al. Enhancement the prediction of postoperative survival in gastric cancer by combining the negative lymph node count with ratio between positive and examined lymph nodes. Ann Surg Oncol 2010;17:1043-51.

17. Coburn NG, Swallow CJ, Kiss A, et al. Significant regional variation in adequacy of lymph node assessment and survival in gastric cancer. Cancer 2006;107:2143-51.

18. Deng J, Zhang R, Pan Y, et al. Comparison of the staging of regional lymph nodes using the sixth and seventh editions of the tumor-node-metastasis (TNM) classification system for the evaluation of overall survival in gastric cancer patients: findings of a case-control analysis involving a single institution in China. Surgery 2014;156:64-74.

19. Deng J, Liang H, Sun D, et al. Prognosis of gastric cancer patients with negative node metastasis following curative resection. Outcomes of the survival and recurrence. Can J Gastroenterol 2008;22:835-9.

20. Wang W, Xu DZ, Li YF, et al. Tumor-ratio-metastasis staging system as an alternative to the 7 th edition UICC TNM system in gastric cancer after D2 resection--results of a single-institution study of 1343 Chinese patients. Ann Oncol 2011;22:2049-56.

21. Sun Z, Xu Y, Li de M, et al. Log odds of positive lymph nodes: a novel prognostic indicator superior to the numberbased and the ratio-based $\mathrm{N}$ category for gastric cancer patients with R0 resection. Cancer 2010;116:2571-80.

22. Deng J, Zhang R, Wu L, et al. Superiority of the ratio between negative and positive lymph nodes for predicting the prognosis for patients with gastric cancer. Ann Surg Oncol 2015;22:1258-66.

23. Rosa F, Tortorelli AP, Alfieri S, et al. Lymph node ratio for gastric cancer: useful instrument or just an expedient to retrieve fewer lymph nodes? Ann Surg 2014;259:e65.

24. Smith DD, Schwarz RR, Schwarz RE. Impact of total lymph node count on staging and survival after gastrectomy for gastric cancer: data from a large USpopulation database. J Clin Oncol 2005;23:7114-24. 
25. Deng JY, Liang H, Sun D, et al. Outcome in relation to numbers of nodes harvested in lymph node-positive gastric cancer. Eur J Surg Oncol 2009;35:814-9.

26. Deng J, Liang H, Sun D, et al. Extended lymphadenectomy improvement of overall survival of gastric cancer patients with perigastric node metastasis. Langenbecks Arch Surg 2011;396:615-23.

Cite this article as: $\mathrm{Gu} \mathrm{P}$, Deng J, Wang W, Wang Z, Zhou Z, Xu H, Liang H. Impact of the number of examined lymph nodes on stage migration in node-negative gastric cancer patients: a Chinese multi-institutional analysis with propensity score matching. Ann Transl Med 2020;8(15):938. doi: 10.21037/ atm-19-4727
27. Zheng WF, Ji TT, Lin Y, et al. The prognostic value of lymph nodes count on survival of patients with nodenegative gastric cancer. Oncotarget 2016;7:43680-8.

28. Huang CM, Lin JX, Zheng CH, et al. Prognostic impact of dissected lymph node count on patients with node-negative gastric cancer. World J Gastroenterol 2009;15:3926-30. 